\title{
Some Thoughts on Transmedia Communication
}

\author{
A. E. HERRMANN ${ }^{1,2}$, VANIA V. ESTRELA ${ }^{3 *}$, HERMES J. LOSCHI ${ }^{4}$ \\ and MIKHAIL P. VISHNEVSKI ${ }^{5}$ \\ 'Department of Romanistic, University of Tuebingen, Tuebingen, Baden-Wuerttemberg, Germany. \\ ${ }^{2}$ Brasilien-Zentrum - Uni Tuebingen, Tuebingen, Baden-Wuerttemberg, Germany. \\ ${ }^{3}$ Telecommunications Department, UFF, RJ, Brazil. \\ ${ }^{4} \mathrm{LCV}$, Faculty of Electrical and Computer Engineering, UNICAMP - Campinas-SP, Brazil. \\ ${ }^{5}$ LCMAT, UENF, Campos de Goytacazes, RJ, Brazil.
}

\author{
(C) \\ Article History \\ Published on 27 November 2018
}

It is hard to define Transmedia (TM) since some communication experts say it is just the best possible way a single story can be understood and followed across different types of media (Figure 1).

It requires adaptation to convey the content from one medium into another. For instance, (i) an interactive book that becomes a movie, and (ii) a comic book that is transformed into a video game.

However, it can have a more drastic meaning as it is the situation when the book ends and a film commences, along with a comic book presenting the protagonist's origin and an accompanying video game reach the market, which is not necessarily a new concept (Figure 2).

A popular, old, and classic example is the Bible during the Dark Ages, when illiteracy abounded and called for stories told in several ways: orally, in plays and told through songs.

There is not a particular motive for the thriving of TM narratives. The further advance of interactive media and the capacity to afford further immersive, participatory practices, accompanied by the demand for original contents from an eventually saturated audience are just a few of the aspects that have added to the TM advance.

TM has applications other than the entertainment industry. Of course, it can expand other facets of the post-modern society, but its implications, contributions, and impacts entail studies.

\section{TM Entertainment and Cultural Areas}

TM consumers do not embrace contents rationally or in other words, what they understand is the

CONTACT Vania V. Estrela $\$ vania.estrela.phd@ieee.org 9 Telecommunications Department, UFF, RJ, Brazil.

\section{(c) (i)}

( 2018 The Author(s). Published by Oriental Scientific Publishing Company

This is an Open Access article licensed under a Creative Commons license: Attribution 4.0 International (CC-BY).

Doi: http://dx.doi.org/10.13005/ojcst11.04.01 
critical message and stores it in their memory. They emotionally embrace the product becoming fans. Storytelling is an excellent way to create a link and to an audience.

TM initiatives are still new and consequently is still a risk-taking task. As a result, audiences feel unique as well as different, and these aspects tend to gather media attention. However, TM is a great engagement mechanism to tell a story in a participative fashion that includes and motivates the audience. This makes TM narratives an exceptional audience consolidator. In entertainment, people can become attracted and passionate about the product they experience. While publicity is perceived as a disruption of what the audience participants want, TM communication can produce high-interest content.

\section{TM in Pedagogy}

Transmedia (TM) storytelling is a powerful constructivist pedagogic tool ${ }^{1,2}$ where the level of engagement offered by multimedia modalities is essential given that no single media fulfill somebody's interest or lifestyle.

Schools have not adopted concrete strategies for this new philosophy, which changes the focus of literacy from being a single individual's expression to become a community asset. It is irrefutable that today's society is a TM globally interconnected realm in which multiple communication platforms are employed. Teaching via a TM storytelling framework involves students interaction with platforms, such as Facebook, YouTube, Pinterest, Instagram, Twitter, or corporative Apps to name a few.

This plethora of platforms allows students' viewpoints, experiences, and resources to create collective intelligence. Such shared nature of multimedia modalities entices, engages, and immerses the individuals, catching their attention. By the way, the attention span of today's students and spectators is smaller than some decades ago.

TM content can also be used by corporations to train employees and managers.

TM training offers the instructors the capacity to lead learners to think critically, sympathize with the material and acquire knowledge, presenting valuable pedagogical context according to the constructivist pedagogy and supporting studentcentered learning. ${ }^{4,5}$ TM descriptions allow for the understanding of the content from the individual's singular perspective, which results in the personalized meaning-making.

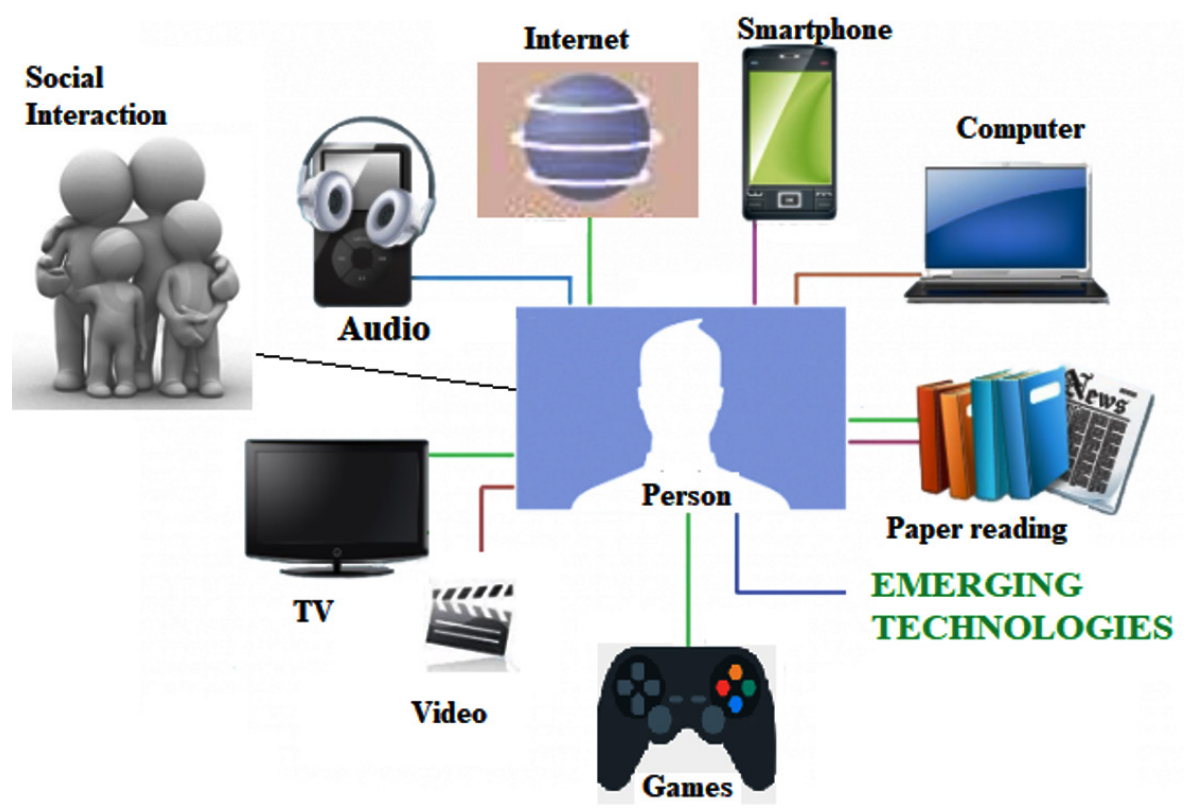

Fig. 1:The Transmedia (TM) Experience 


\section{TM in Healthcare}

Perhaps, the most beneficial use of TM technology is in the healthcare domain, since it can help psychotherapy, rehabilitation, physiotherapy, development of social and communication skills, occupational therapy, geriatrics, and sports medicine among other fields.

The use of ICT technologies such as video games as therapeutic tools in rehabilitation for stroke patients, wheelchair users, people living with cerebral palsy, chemotherapy patients, muscular dystrophy sufferers, and to treat autism ${ }^{6,8}$ can amplify the TM scope as far as applications go. ICT can also help to formulate new procedures to better handle contexts, which still remains an essential concern.

\section{Essential Aspects of TM Design and Deployment} Current TM development uses the following model: initiatives are presented to somebody in one division of the company and undergoes a somewhat messy workflow. Hence, the best projects come from either (i) well-established companies, or (ii) very creative, although indie, independent producers.

Avoiding spoilers, introduced people from outside the target group can also be a significant concern. Below, some crucial aspects of TM design and deployment are discussed.

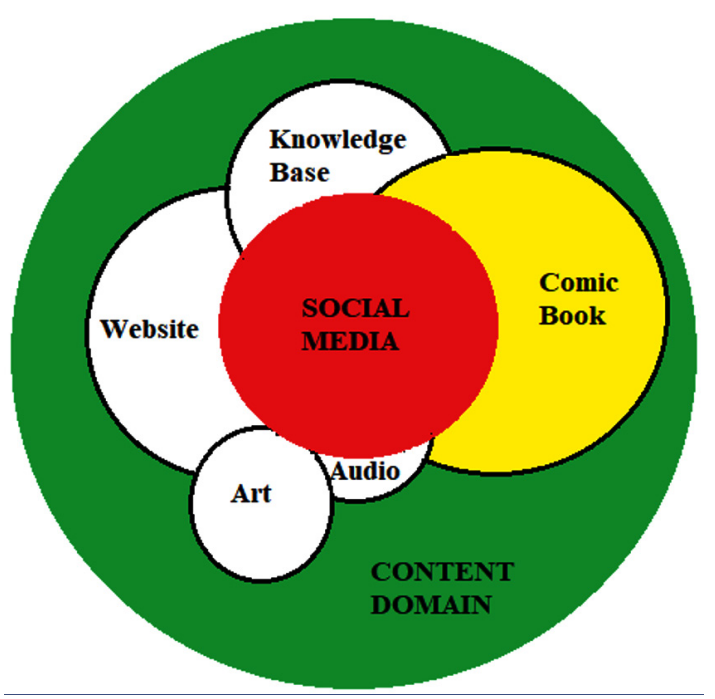

Fig. 2: Interaction Among Different Media Modalities

\section{TM Depth}

The key is to design a TM product well. It is not about ruining a narrative, splitting and organizing it at more or less random topics. Its parts must handle maximum significance from each medium and provide a micro-involvement that motivates the audience to know more.

A TM project is entirely different from a conventional implementation of content. As an alternative to repeating the same item repeatedly in as many different media as conceivable, each new contact point must have a different influence to transport the audience through different depth levels. Each new impact enhances the experience before it and the message as a whole.

\section{TM True Integration}

A TM experience demands true integration: all the portions contribute to a single piece so that a story is only completely assimilated when lived as a whole (Figure 3).

To produce as well as to distribute content among all the parts is not enough. Each content creating subgroup involved must trade their autonomy in favor of genuine collaboration.

\section{Identification of Possible TM Participatory Experiences}

All TM actions necessitate that the participation of the audience, since they must follow a narrative

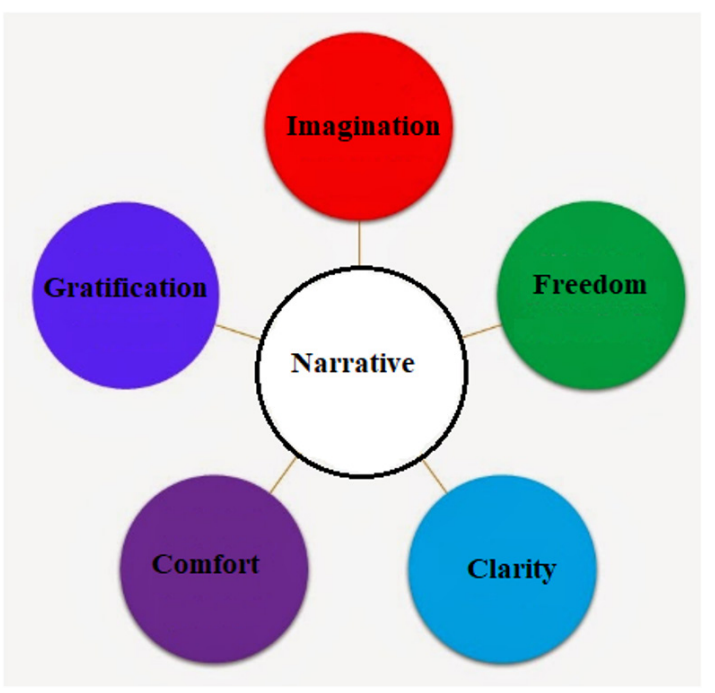

Fig. 3: TM Engagement Elements 
articulated through different media, ranging from printed matter to a video, web radio or a theater performance. Some may require specific cues from the viewers to move forward (Figure 3). Nevertheless, not all TM projects are suitable for interactive implementations.

There are some TM actions where the audience is a passive matter to the action like thrillers that alternate between orthodox reading and online video that develop upon the central narrative.

On the other hand, other TM forms are participatory such as Alternate Reality Games (ARGs) where something takes place if the audience unravels mysteries, visits particular places and perform particular activities, either alone or in a group.

\section{TM Suitability}

TM operations capture and engender audience fidelity, but there are other methods. The idea of immersion and capture by a narrative does not appeal to every person, which is why TM operations need an adequate audience segmentation.

Furthermore, TM does not work if the intent is merely to inform without offers and promotions. Targeted content is built around principles, opinions, and points of view, but not grounded on exclusive intentions. Hence, it is paramount to identify the main set of intrinsic characteristics of current stage of the narrative that is suitable to be made into a TM story.

\section{TM Cost}

Any TM endeavor is costly regarding time, staffs and money. Therefore, the financial impact of TM is a significant concern. Most TM operations relate to a business model that recognizes TM as a way for encouraging and promoting something else, rather than the way content will be delivered. That is how TM methodologies arose, and as a result, there is an unforeseen financial impact through media treatment and the publicity it generates. Fidelity and trust issues should also come to play.

A target group may rely on Self-financed TM (SFTM) where an audience is willing to pay for some experience.

\section{Flexible Interface Development}

In this era of TM discourse and Postmedia statements, talking about medium specificity is still productive, given that novel media forms or modalities are replacing others rapidly and without enough time to thoroughly explore their potentials. An exchange of ideas about medium specificity will enable us to improve unique possibilities that otherwise can be overlooked.

\section{Conclusion}

More studies are needed to investigate where TM differs is in the communication ecosystem, as this is created as an entire piece, with the different threads positioned strategically, for a maximum immersion as well as sensory experience., ${ }^{4,6}$

Study-training material using TM can boost training and provokes learners in a more profound and longer lasting way than multimedia alone.

The target group reports most of the time, the sensation of being transported by the content to another world.

In many ways, the media elements are not fundamentally different from other media elements. It is all in the way the content is organized and adapted to a suitable TM model where every part fits together.

\section{References}

1. Jenkins $\mathrm{H}$. Storytelling and Entertainment: An Annotated Syllabus, Continuum: Journal of Media \& Cultural Studies, 24:6, 943-958 2010.

2. Pratten, R.(2015). Getting Started in Transmedia Storytelling: A Practical Guide for Beginners(2nd ed.). London, UK:
CreateSpace. p.224. ISBN 978-1-5153-39168.

3. Bernardo, N. (2011). The Producers Guide to Transmedia: How to Develop, Fund, Produce and Distribute Compelling Stories Across Multiple Platforms (Paperback). London, UK: beActive Books. p.153. ISBN 978-0-9567500- 
0-6.

4. Kinder, M., McPherson, T. (Eds.). (2014). Transmedia Frictions: The Digital, the Arts, and the Humanities. University of California Press. Retrieved from http://www.jstor.org/ stable/10.1525/j.ctt6wqc2f.

5. http://www.thebcma.info/transmediastorytelling/Retrieved on October 12, 2018.

6. Griffiths, M., Kuss, D. J., Ortiz de Gortari, A. B. (2013). Videogames as Therapy: A Review of the Medical and Psychological Literature. In M. Cruz-Cunha, I. Miranda, \& P. Gonçalves (Eds.), Handbook of Research on ICTs and Management Systems for Improving Efficiency in Healthcare and Social Care (pp. 43-68). Hershey, PA: IGI Global. doi:10.4018/978-1-4666-3990-4.ch003.

7. Warren, S., Wakefield, J.S., Mills, L. Learning and Teaching as Communicative Actions: Transmedia Storytelling, in Laura A. Wankel, Patrick Blessinger (ed.) Increasing Student Engagement and Retention using Multimedia Technologies: Video Annotation, Multimedia Applications, Videoconferencing and Transmedia Storytelling (Cuttingedge Technologies in Higher Education, Volume 6), 2013, Emerald Group Publishing Limited, pp.67-94 doi:10.1108/S20449968(2013)000006F006.

8. Rittmann, Tim (April 2013). Wer das Game spielt und die Serie sieht, hat mehr davon. ZEIT Online. Retrieved on October 122017. 